\title{
Constructing large peak-pit Condorcet domains
}

\author{
Alexander Karpov ${ }^{1,2} \cdot$ Arkadii Slinko $^{3}$ \\ Accepted: 13 December 2021 / Published online: 23 February 2022 \\ (C) The Author(s) 2022
}

\begin{abstract}
We present a new method of constructing Condorcet domains from pairs of Condorcet domains of smaller sizes (concatenation + shuffle scheme). The concatenation + shuffle scheme provides maximal, connected, copious, peak-pit domains whenever the original domains have these properties. It allows to construct maximal peak-pit Condorcet domains that are larger than those obtained by the Fishburn's alternating scheme for all $n \geq 13$ alternatives. For a large number $n$ of alternatives, we get a lower bound $2.1045^{n}$ for the cardinality of the largest peak-pit Condorcet domain and a lower bound $2.1890^{n}$ for the cardinality of the largest Condorcet domain, improving Fishburn's result. We also show that all Arrow's single-peaked domains can be constructed by concatenation + shuffle scheme starting from the trivial domain.
\end{abstract}

Keywords Condorcet domain · Never conditions · Peak-pit domain · DanilovKarzanov-Koshevoy construction · Arrow's single-peaked domain

\section{Introduction}

The famous Condorcet Paradox shows that if voters' preferences are unrestricted, the majority voting can lead to intransitive collective preference, in which case the majority rule introduced by Condorcet (1785), despite all its numerous advantages, is unable to determine the best alternative, i.e. it is not always decisive. Arrow (1963) introduced the concept of a universal domain and domain restrictions emphasising the importance of the latter. He argued that in many instances, voters

\footnotetext{
$\triangle$ Arkadii Slinko

a.slinko@auckland.ac.nz

Alexander Karpov

akarpov@hse.ru

1 Department of Economics, HSE University, Moscow, Russia

2 Institute of Control Sciences, Russian Academy of Sciences, Moscow, Russia

3 Department of Mathematics, University of Auckland, Auckland, New Zealand
} 
or economic agents cannot have arbitrary preferences. In particular, the condition of single-peaked preferences Black (1948), which prevents Condorcet paradox, was especially useful in political science modelling voters on the one-dimensional leftright political spectrum. Domain restrictions is nowadays an important topic in economics and computer science alike Elkind (2018). In particular, for artificial societies of autonomous software agents there is no problem of individual freedom and, hence, for the sake of having transitive collective decisions, the designer can restrict choices of those artificial agents to make the majority rule work every time.

Condorcet domains represent an ultimate solution to this problem as they are sets of linear orders with the property that, whenever the preferences of all voters belong to this set, the majority relation of any profile with an odd number of voters is transitive. In particular, the domain of single-peaked preferences is one of the Condorcet domains. Maximal Condorcet domains historically have attracted a special attention since they represent a compromise which allows a society to always have transitive collective preferences and, under this constraint, provide voters with as much individual freedom as possible. However, maximal Condorcet domains may have different cardinalities which can be as low as four Danilov and Koshevoy (2013). Thus, the question: "How large a Condorcet domain can be?" has attracted even more attention (see the survey of Monjardet (2009) for a fascinating account of historical developments). Kim et al. (1992) identified this problem as a major unsolved problem in the mathematical social sciences.

Fishburn (1996a) addressing this question introduced the function

$$
f(n)=\max \{|\mathcal{D}|: \mathcal{D} \text { is a Condorcet domain on a set of } n \text { alternatives }\} .
$$

and put this problem in the mathematical perspective asking for the maximal values of this function to be found or at least estimated.

Abello (1991) and Fishburn (1996a, 2002) managed to construct some "large" Condorcet domains based on different ideas. Fishburn, in particular, taking a clue from Monjardet example (sent to him in a private communication), came up with the so-called alternating scheme domains, later called Fishburn's domains Danilov and Karzanov (2012). This scheme produces Condorcet domains with some nice properties, which, in particular, are connected and have maximal width (these concepts will be defined later in the paper). Fishburn (1996a) conjectured (Conjecture 2) that among Condorcet domains that, for any triple of alternatives, do not satisfy the so-called never-middle condition (these in Danilov and Karzanov (2012) were later called peak-pit domains), the alternating scheme provides domains of maximum cardinality. Labbé and Lange (2020) showed that Fishburn's domain has the largest size among domains associated with the weak order of a finite Coxeter group. The focus of attention on maximal peak-pit domains was justified by their important connections to such classical combinatorial objects as rhombus tilings, arrangements of pseudolines and maximal separated set systems (Leclerc and Zelevinsky 1998; Galambos and Reiner 2008; Danilov and Karzanov 2012).

In early work, Condorcet domains on the set of $n$ alternatives were usually required to have maximal width which (up to an isomorphism) means that they must 
contain two completely reversed linear orders $12 \ldots n$ and $n . .21$. Monjardet (2006) introduced (in slightly different terms) the function

$$
\begin{aligned}
g(n)= & \max \{|\mathcal{D}|: \mathcal{D} \text { is a a peak-pit Condorcet domain of maximal width } \\
& \text { on a set of } n \text { alternatives }\} .
\end{aligned}
$$

It was hypothesised Fishburn (1996a) that $g(n)=\left|F_{n}\right|$, which means that Fishburn's domains are the largest in the class of peak-pit Condorcet domain of maximal width.

Recently, the necessity of the requirement of maximal width was questioned for a number of reasons. In particular, Arrow's single-peaked domain (which is a Condorcet domain whose restriction on each triple of alternatives is single-peaked (Arrow 1963)) is a very natural class of Condorcet domains without this requirement (Slinko 2019). In addition, not every society is liberal enough to allow completely opposite views. Thus, we introduce another function

$$
h(n)=\max \{|\mathcal{D}|: \mathcal{D} \text { is a peak-pit domain on a set of } n \text { alternatives }\} .
$$

It is known that $f(n)=g(n)=h(n)$ for $n \leq 7$ (Fishburn 1996a; Galambos and Reiner 2008) and it was believed that $g(16)<f(16)$ (Monjardet 2009). This is because Fishburn (1996a) showed that $f(16)>\left|F_{16}\right|$. Thus, if Fishburn's hypothesis was true, we would get $f(n)>g(n)$ for $n \geq 16$. However, this hypothesis appeared to be false.

Danilov and Karzanov (2012) introduced the class of tiling domains which are peak-pit domains of maximal width and defined an operation of concatenation on tiling domains that allowed them to show that $g(42)>\left|F_{42}\right|$ refuting Fishburn's conjecture. In the present article, we give an algebraic definition, a generalisation, and an extension of the Danilov-Karzanov-Koshevoy construction (abbreviated DKK-construction), which we call concatenation + shuffle scheme, and investigate its properties.

In our interpretation, the DKK-construction $\otimes_{1}$ involves two peak-pit Condorcet domains $\mathcal{D}_{1}$ and $\mathcal{D}_{2}$ on sets of $n$ and $m$ alternatives, respectively, and two linear orders $u \in \mathcal{D}_{1}$ and $v \in \mathcal{D}_{2}$; the result is denoted as $\left(\mathcal{D}_{1} \otimes_{1} \mathcal{D}_{2}\right)(u, v)$. It is again a peak-pit Condorcet domain on $n+m$ alternatives whose exact cardinality we can calculate. A drawback of the DKK-construction is that when $\mathcal{D}_{1}$ and $\mathcal{D}_{2}$ are maximal Condorcet domains, $\left(\mathcal{D}_{1} \otimes_{1} \mathcal{D}_{2}\right)(u, v)$ may not be maximal. We fix this shortcoming by introducing a new construction $\left(\mathcal{D}_{1} \otimes_{2} \mathcal{D}_{2}\right)(u, v)$ that always contains $\left(\mathcal{D}_{1} \otimes_{1} \mathcal{D}_{2}\right)(u, v)$ and is always maximal. It allows us to prove the inequality $h(13)>\left|F_{13}\right|$. Using the new construction, we can also show that $g(34)>\left|F_{34}\right|$ further improving the result of Danilov and Karzanov (2012).

We also prove that for large $n$, we have lower bounds $g(n) \geq 2.0767^{n}$, $h(n) \geq 2.1045^{n}$ and $f(n) \geq 2.1890^{n}$, where the first confirms the unpublished result of Ondrej Bilka announced in Felsner and Valtr (2011) and the third improves Fishburn's (1996a) bound while the second result is completely new. We also get an upper bound $g(n)<2.4870^{n}$ for function $g$. For other functions, it is only known that they are bounded by $c^{n}$ for some constant $c$.

Concatenation + shuffle scheme reveals the structural properties and generation algorithm for Arrow's single-peaked domains. We show that all Arrow's single- 
peaked domains can be constructed by concatenation + shuffle scheme starting from the trivial domain.

The paper is organised as follows. Section 2 contains preliminaries, Sect. 3 gives the algebraic description and properties of the original Danilov-KarzanovKoshevoy construction and proves the lower bound for function $g$. Section 4 introduces the concatenation + shuffle scheme, studies its properties and proves that all Arrow's single-peaked domains can be obtained iteratively using the concatenation + shuffle scheme from the trivial domain. Section 5 is devoted to construction of Condorcet domains of cardinality larger than Fishburn's domains. It also contains a new bound for function $h$ and improved bound for function $f$. A short Sect. 6 outlines what is known in relation to upper bounds, most notably, an upper bound for function $g$, and Sect. 7 concludes.

\section{Preliminaries}

Let $A$ be a finite set and $\mathcal{L}(A)$ be the set of all (strict) linear orders on $A$. Any subset $\mathcal{D} \subseteq \mathcal{L}(A)$ will be called a domain. Any sequence $P=\left(v_{1}, \ldots, v_{n}\right)$ of linear orders from $\mathcal{D}$ will be called a profile ${ }^{1}$ over $\mathcal{D}$. It usually represents a collective set of opinions of a society about merits of alternatives from $A$. A linear order $a_{1}>a_{2}>\cdots>a_{n}$ on $A$, will be denoted by a string $a_{1} a_{2} \cdots a_{n}$. Let us also introduce notation for reversing orders: if $x=a_{1} a_{2} \cdots a_{n}$, then $\bar{x}=a_{n} a_{n-1} \cdots a_{1}$. If linear order $v$ ranks $a$ higher than $b$, we denote this as $a \succ_{v b}$.

Definition 1 The majority relation $\succeq_{P}$ of a profile $P=\left(\succ_{1}, \ldots, \succ_{n}\right)$ is defined as

$$
a \succeq_{P} b \Longleftrightarrow\left|\left\{i \mid a \succ_{i} b\right\}\right| \geq\left|\left\{i \mid b \succ_{i} a\right\}\right| .
$$

Verbally, $a \succeq_{P} b$ means that at least as many voters from a society with profile $P$ prefer $a$ to $b$ as voters who prefer $b$ to $a$. For an odd number of linear orders in the profile $P$, this relation is a tournament, i.e. complete and asymmetric binary relation. In this case, we denote the majority relation as $\succ_{P}$.

Now, we can define the main object of this investigation.

Definition 2 A domain $\mathcal{D} \subseteq \mathcal{L}(A)$ over a set of alternatives $A$ is a Condorcet domain if the majority relation $\succ_{P}$ of any profile $P$ over $\mathcal{D}$ with an odd number of voters is transitive. A Condorcet domain $\mathcal{D}$ is maximal if for any Condorcet domain $\mathcal{D}^{\prime} \subseteq \mathcal{L}(A)$ the inclusion $\mathcal{D} \subseteq \mathcal{D}^{\prime}$ implies $\mathcal{D}=\mathcal{D}^{\prime}$

There is a number of alternative definitions of Condorcet domains, see, e.g. Monjardet (2009) and Puppe and Slinko (2019).

The same Condorcet domain can be presented in different 'disguises' so we need to recognise when this happens. For this, we need the following concepts. Let $\psi: A \rightarrow A^{\prime}$ be a bijection between two sets of alternatives $A$ and $A^{\prime}$. It can then be extended to a mapping $\psi: \mathcal{L}(A) \rightarrow \mathcal{L}\left(A^{\prime}\right)$ (we will use the same letter to denote

\footnotetext{
${ }_{1}$ A profile, unlike the domain, can have several identical linear orders.
} 
both) in two ways: by mapping a linear order $u=a_{1} a_{2} \cdots a_{m}$ onto $\psi(u)=$ $\psi\left(a_{1}\right) \psi\left(a_{2}\right) \cdots \psi\left(a_{m}\right)$ or onto $\overline{\psi(u)}=\psi\left(a_{m}\right) \psi\left(a_{m-1}\right) \cdots \psi\left(a_{1}\right)$.

Definition 3 Let $A$ and $A^{\prime}$ be two sets of alternatives (not necessarily distinct) of equal cardinality. We say that two domains, $\mathcal{D} \subseteq \mathcal{L}(A)$ and $\mathcal{D} \subseteq \mathcal{L}\left(A^{\prime}\right)$ are isomorphic if there is a bijection $\psi: A \rightarrow A^{\prime}$ such that $\mathcal{D}^{\prime}=\{\psi(d) \mid d \in \mathcal{D}\}$ and flip-isomorphic if $\mathcal{D}^{\prime}=\{\overline{\psi(d)} \mid d \in \mathcal{D}\}$.

Up to an isomorphism, there is only one maximal Condorcet domain on the set $\{a, b\}$, namely $C D_{2}=\{a b, b a\}$ and there are only three maximal Condorcet domains on the set $\{a, b, c\}$, namely

$$
\begin{gathered}
\mathrm{CD}_{3, t}=\{a b c, a c b, c a b, c b a\}, \quad \mathrm{CD}_{3, m}=\{a b c, b c a, a c b, c b a\}, \\
\mathrm{CD}_{3, b}=\{a b c, b a c, b c a, c b a\} .
\end{gathered}
$$

The first domain contains all the linear orders on $a, b, c$ where $b$ is never ranked first, the second domain contains all the linear orders on $a, b, c$ where $a$ is never ranked second and the third domain contains all the linear orders on $a, b, c$ where $b$ is never ranked last. Following Monjardet, we denote these conditions as $b N_{\{a, b, c\}} 1$, $a N_{\{a, b, c\}} 2$ and $b N_{\{a, b, c\}} 3$, respectively, and call never conditions. We note that these are the only conditions of type $x N_{\{a, b, c\}} i$ with $x \in\{a, b, c\}$ and $i \in\{1,2,3\}$ that these domains satisfy.

All three domains $C D_{3, t}, C D_{3, m}, C D_{3, b}$ are not isomorphic but the first and the third ones are flip-isomorphic under the identity mapping of $\{a, b, c\}$ onto itself.

A domain that for any triple $a, b, c \in A$ satisfies a condition $x N_{\{a, b, c\}} 1$ with $x \in$ $\{a, b, c\}$ is called never-top domain, a domain that for any triple $a, b, c \in A$ satisfies a condition $x N_{\{a, b, c\}} 2$ with $x \in\{a, b, c\}$ is called never-middle domain, and a domain that for any triple $a, b, c \in A$ satisfies a condition $x N_{\{a, b, c\}} 3$ with $x \in$ $\{a, b, c\}$ is called never-bottom domain or Arrow's single-peaked domain (Slinko 2019). However, most commonly, a Condorcet domain for different triples of alternatives satisfies different set of never conditions. We need to restrict the types of never conditions to have a meaningful theory.

Definition 4 (Danilov and Karzanov 2012) A domain that for any triple satisfies either never-top or never-bottom condition is called a peak-pit domain. Both nevertop and never-bottom conditions will be called peak-pit conditions.

We note that Arrow's single-peaked domains, single-crossing domains and Fishburn's domains are all peak-pit domains.

Definition 5 (Puppe 2018) A Condorcet domain $\mathcal{D}$ is said to have maximal width if it contains two completely reversed orders, i.e. together with some linear order $u$ it also contains its flip $\bar{u}$.

Up to an isomorphism, for any Condorcet domain $\mathcal{D}$ of maximal width we may assume that $A=[n]=\{1,2 \ldots, n\}$ and it contains linear orders $e=12 \cdots n$ and $\bar{e}=n \cdots 21$. 
We note that Danilov and Karzanov (2012), who consider linear orders over $A=[n]$, restrict in their investigation the class of peak-pit domains to domains of maximal width. They prove that under this restriction all peak-pit domains can be embedded into tiling domains (Theorem 2 of Danilov and Karzanov (2012)).

Early research similarly concentrated on Condorcet domains of maximal width. However, we note that most maximal Arrow's single-peaked domains do not satisfy the maximal width requirement (Slinko 2019).

Given a set of alternatives $A$, we say that

$$
\mathcal{N}=\left\{x N_{\{a, b, c\}} i \mid\{a, b, c\} \subseteq A, x \in\{a, b, c\} \text { and } i \in\{1,2,3\}\right\}
$$

is a complete set of never conditions if it contains at least one never condition for every triple $a, b, c$ of distinct elements of $A$.

Proposition 1 A domain of linear orders $\mathcal{D} \subseteq \mathcal{L}(A)$ is a Condorcet domain if and only if it is non-empty and satisfies a complete set of never conditions.

Proof This is well-known characterisation noticed by many researchers. See, for example, Theorem 1(d) in Puppe and Slinko (2019) and references there.

This proposition, in particular, means that the collection $\mathcal{D}(\mathcal{N})$ of all linear orders that satisfy a certain complete set of never conditions $\mathcal{N}$, if non-empty, is a Condorcet domain.

Permutohedron $^{2}$ for the purposes of this paper is a graph whose vertexes are labelled by the permutations of the first $n$ natural numbers that can be, in turn, identified with the linear orders on $[n]$. The edges connect vertices corresponding to linear orders that differ by a swap of neighbouring alternatives.

A pair $(i, j)$ is called an inversion of a linear order $u \in \mathcal{L}([n])$ if $i<j$ but $j \succ_{u} i$. The set of inversions of order $u$ is denoted by $\operatorname{Inv}(u)$. For linear orders $u, v \in \mathcal{L}([n])$, we write $u \ll v$ if $\operatorname{Inv}(u) \subseteq \operatorname{Inv}(v)$. The relation $\ll$ is called the weak Bruhat order, and the partially ordered set $(\mathcal{L}([n]), \ll)$ is called the Bruhat poset. ${ }^{3}$

A linear order $u$ covers a linear order $v$ if $\operatorname{Inv}(u)$ equals $\operatorname{Inv}(v)$ plus exactly one inversion (this is known to agree with the notion of covering in a poset). The Bruhat digraph is formed by drawing a directed edge from $u$ to $v$ if and only if $v$ covers $u$, in which case $u$ and $v$ are connected by an edge in the permutohedron. A maximal chain in the Bruhat digraph connects $e=12 \cdots n$ with $\bar{e}=n \cdots 21$. Danilov and Karzanov (2012) called a domain of maximal width semi-connected if it contains such a chain.

The permutohedron is also naturally endowed with the following betweenness structure (as defined by Kemeny (1959)). An order $v$ is between orders $u$ and $w$ if $v \supseteq u \cap w$, i.e. $v$ agrees with all binary comparisons in which $u$ and $w$ agree (see also Kemeny and Snell (1960)). The set of all orders in $\mathcal{L}([n])$ that are between $u$ and $w$ is called the interval spanned by $u$ and $w$ and is denoted by $[u, w]$.

\footnotetext{
${ }^{2}$ Permutohedron of order $n$ is an $(n-1)$-dimensional polytope embedded in an $n$-dimensional space. The graph that we consider is also called the skeleton of the permutohedron.

3 Monjardet (2009) calls it permutoèdre lattice.
} 
Given a domain of preferences $\mathcal{D} \subseteq \mathcal{L}([n])$, for any $u, w \in \mathcal{D}$, we define the induced interval as $[u, w]_{\mathcal{D}}=[u, w] \cap \mathcal{D}$. Puppe and Slinko (2019) defined a graph $G_{\mathcal{D}}$ associated with the domain $\mathcal{D}$. The set of linear orders from $\mathcal{D}$ are the set of vertices $V_{\mathcal{D}}$, and for two orders $u, w \in \mathcal{D}$ we draw an edge between them if there is no other vertex between them, i.e. $[u, w]_{\mathcal{D}}=\{u, w\}$. The set of edges is denoted $E_{\mathcal{D}}$ so the graph is $G_{\mathcal{D}}=\left(V_{\mathcal{D}}, E_{\mathcal{D}}\right)$. As established in Puppe and Slinko (2019), for any Condorcet domain $\mathcal{D}$ the graph $G_{\mathcal{D}}$ is a median graph (Mulder 1978) and any median graph can be obtained in this way. A domain $\mathcal{D}$ is called connected if its graph $G_{\mathcal{D}}$ is a subgraph of the permutahedron (Puppe and Slinko 2019); we note that domains $C D_{3, t}$ and $C D_{3, b}$ are connected but $C D_{3, m}$ is not.

Danilov and Karzanov (2012) proved that a maximal Condorcet domain of maximal width is peak-pit domain if and only if it is semi-connected. Puppe (2016) showed that for a maximal Condorcet domain semi-connectedness implies direct connectedness (Proposition A2) which means that any two linear orders in the domain are connected by a shortest possible (geodesic) path.

A rhombus tiling (or simply a tiling) is a subdivision $T$ into rhombic tiles of a zonogon $Z(n ; 2)$ formed by the points $\sum_{i} a_{i} \psi_{i}$, where $0 \leq a_{i} \leq 1$ and $\psi_{1}, \ldots, \psi_{n}$ are unit vectors in the upper half-plane. This centre-symmetric $2 n$-gon has the origin as its bottom vertex $b$ and the top vertex $t=\psi_{1}+\cdots+\psi_{n}$. An $i j$-tile is a rhombus congruent to the one formed by the points $a \psi_{i}+b \psi_{j}$, where $0 \leq a, b \leq 1$. A snake is a path from $b$ to $t$ along the boundaries of the tiles which for each $i=1, \ldots, n$ contains a unique segment parallel to $\psi_{i}$. Each snake corresponds to a linear order on $\{1, \ldots, n\}$ in the following way. If a point travelling from $b$ to $t$ passes segments parallel to $\psi_{i_{1}}, \psi_{i_{2}} \ldots, \psi_{i_{n}}$, then the corresponding linear order will be $i_{1} i_{2} \cdots i_{n}$. The set of snakes of a rhombus tiling, thus, defines a domain which is called a tiling domain. Danilov and Karzanov (2012) showed that maximal peak-pit domains of maximal width are exactly the tiling domains (see an example on Fig. 1). Due to

Fig. 1 Median graph and the tiling of the hexagon for Fishburn's domain $F_{4}$

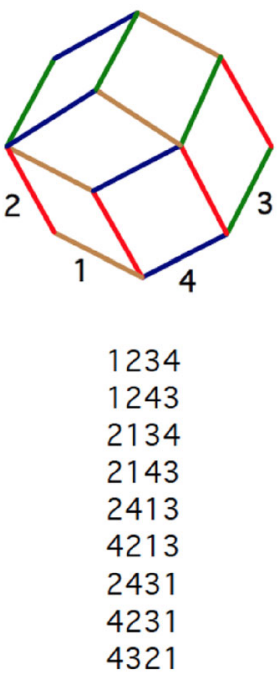

4213

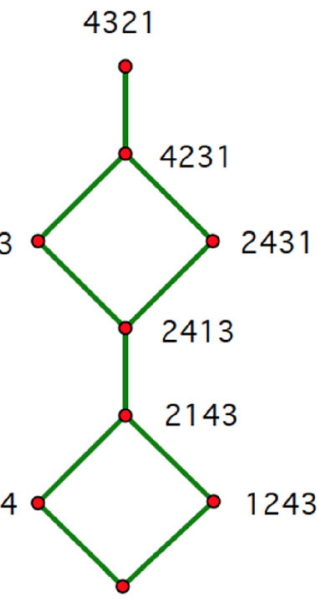

1234 
equivalence of rhombus tilings and arrangements of pseudolines (Elnitsky 1997; Felsner 2012), this also implies that peak-pit domains of maximal width can be associated with the set of flags in the set of chamber sets of arrangements of pseudolines (Li et al. 2021).

Finally, we give two more definitions that express two properties of Condorcet domains. But, first, we will introduce the following notation. Suppose $\mathcal{D} \subseteq \mathcal{L}(A)$ be a domain on the set $A$ and let $B \subseteq A$. Suppose also $u \in \mathcal{D}$. Then, by $\mathcal{D}_{B}$ and $u_{B}$ we denote the restrictions of $\mathcal{D}$ and $u$ onto $B$, respectively. In particular, $\mathcal{D}_{B} \subseteq \mathcal{L}(B)$ and $u_{B} \in \mathcal{L}(B)$.

Definition 6 We call a Condorcet domain $\mathcal{D}$ ample if for any pair of alternatives $a, b \in A$ the restriction $\mathcal{D}_{\{a, b\}}$ of this domain to $\{a, b\}$ has two distinct orders, that is, $\mathcal{D}_{\{a, b\}}=\{a b, b a\}$.

Definition 7 (Slinko 2019) A Condorcet domain $\mathcal{D}$ is called copious if for any triple of alternatives $a, b, c \in A$ the restriction $\mathcal{D}_{\{a, b, c\}}$ of this domain to this triple has four distinct orders, that is, $\left|\mathcal{D}_{\{a, b, c\}}\right|=4$.

Of course, any copious Condorcet domain is ample. We note that if a Condorcet domain $\mathcal{D}$ is copious, then it satisfies a unique complete set of never conditions.

Definition 8 Let $A=[n]$. A complete set of peak-pit conditions (1) is said to satisfy the alternating scheme (Fishburn 1996a), if for all $1 \leq i<j<k \leq n$, it includes never conditions

$$
j N_{\{i, j, k\}} 3 \text {, if } j \text { is even, and } j N_{\{i, j, k\}} 1 \text {, if } j \text { is odd }
$$

or

$$
j N_{\{i, j, k\}} 1 \text {, if } j \text { is even, and } j N_{\{i, j, k\}} 3 \text {, if } j \text { is odd. }
$$

The domains of linear orders on $[n]$ that are determined by these complete sets we denote $F_{n}$ and $\overline{F_{n}}$, respectively, and call Fishburn's domains (Danilov and Karzanov 2012). The second domain is flip-isomorphic to the first so we usually consider only the first one. In particular, $F_{2}=\{12,21\}, F_{3}=\{123,213,231,321\}$ and

$$
F_{4}=\{1234,1243,2134,2143,2413,2431,4213,4231,4321\} .
$$

Figure 1 shows the median graph of $F_{4}$ and its representation as a tiling domain. Galambos and Reiner (2008) gave the exact formula for the cardinality of $F_{n}$ : 


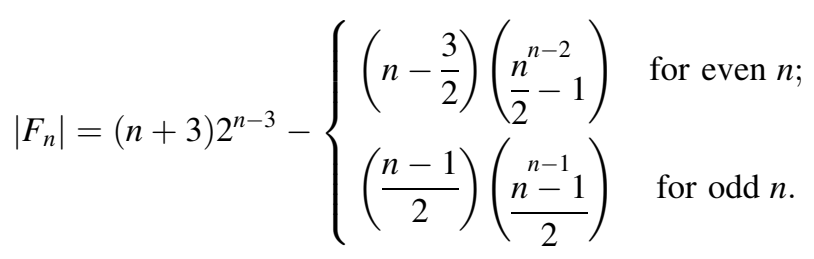

In a semi-connected domain, the order $12 \cdots n$ can be transformed into $n \cdots 21$ by a sequence of swaps of neighboring alternatives so that each pair is switched exactly once. If $i<j<k$, then there are two possible ways in which $i j k$ is converted into $k j i$ during this transformation, namely

$$
i j k \rightarrow j i k \rightarrow j k i \rightarrow k j i \quad \text { and } \quad i j k \rightarrow i k j \rightarrow k i j \rightarrow k j i .
$$

In the second case, the triple $[i, j, k]$ is called an inversion. Galambos and Reiner (2008) showed that if a maximal Condorcet domain $\mathcal{D}$ of maximal width contains one maximal chain in the Bruhat digraph (i.e. it is semi-connected), then $\mathcal{D}$ is a union of all maximal chains that are equivalent to the given one in the sense that those maximal chains satisfy the same set of inversion triples. Thus, any maximal semi-connected Condorcet domain $\mathcal{D}$ can be defined by the set of inversion triples. In particular, the domain $F_{4}$ can be defined by the set of inversion triples

$$
\{[1,3,4],[2,3,4]\} \text {. }
$$

This provides a compact characterisation for semi-connected Condorcet domains.

\section{Algebraic description and properties of the Danilov-Karzanov- Koshevoy construction}

Danilov and Karzanov (2012) define the 'concatenation' of two tiling domains by the picture shown in Fig. 2 (where one arrow is obviously missing but it is present in the arXiv.org version (Danilov et al. 2011).

Let us now start describing this construction algebraically. In fact, this will be a generalisation of their construction since in our construction two arbitrary linear orders are additionally involved. First, we describe the 'pure' concatenation.

Let $\mathcal{D}_{1}$ and $\mathcal{D}_{2}$ be two Condorcet domains on disjoint sets of alternatives $A$ and $B$, respectively. We define a concatenation of these domains as the domain

$$
\mathcal{D}_{1} \odot \mathcal{D}_{2}=\left\{x y \mid x \in \mathcal{D}_{1} \text { and } y \in \mathcal{D}_{2}\right\}
$$

on $A \cup B$. It is immediately clear that $\mathcal{D}_{1} \odot \mathcal{D}_{2}$ is also a Condorcet domain of cardinality $\left|\mathcal{D}_{1} \odot \mathcal{D}_{2}\right|=\left|\mathcal{D}_{1}\right|\left|\mathcal{D}_{2}\right|$. We have only to check that one of the never conditions is satisfied for each triple $\left\{a_{1}, a_{2}, b\right\}$ where $a_{1}, a_{2} \in A$ and $b \in B$ (for triples $\left\{a, b_{1}, b_{2}\right\}$ the argument will be similar). The restriction $\left(\mathcal{D}_{1} \odot \mathcal{D}_{2}\right)_{\left\{a_{1}, a_{2}, b\right\}}$ will contain at most two linear orders $a_{1} a_{2} b$ and $a_{2} a_{1} b$, which is consistent with both never-top and never-bottom conditions. This domain corresponds to the snakes lying entirely within $T$ and $T^{\prime}$ on Fig. 2. 
Fig. 2 Concatenation of tilings $T$ and $T^{\prime}$

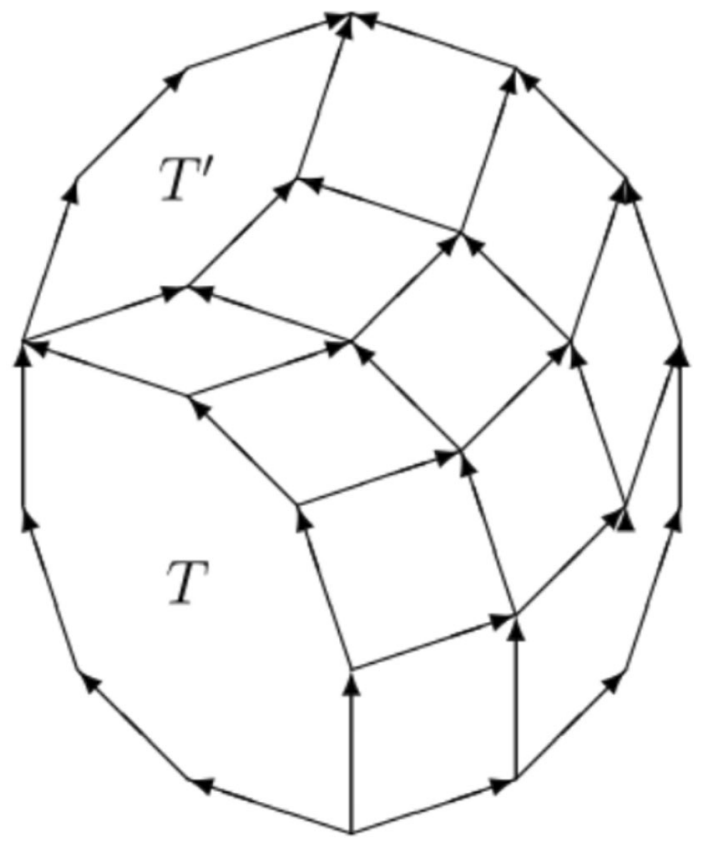

Definition 9 Let $A$ and $B$ be two disjoint sets of alternatives, $u \in \mathcal{L}(A)$ and $v \in \mathcal{L}(B)$. An order $w \in \mathcal{L}(A \cup B)$ is said to be a shuffle of $u$ and $v$ if $w_{A}=u$ and $w_{B}=v$, i.e. the restriction of $w$ onto $A$ is equal to $u$ and the restriction of $w$ onto $B$ is equal to $v$.

For example, 516723849 is a shuffle of 1234 and 56789.

Given two linear orders $u$ and $v$, we define domain $u \oplus v$ on $A \cup B$ as the set of all shuffles of $u$ and $v$. It is clear from the definition that $u \oplus v=v \oplus u$. The cardinality of this domain is $|u \oplus v|=\left(\begin{array}{c}n+m \\ m\end{array}\right)$. We believe this domain corresponds to what is depicted in Fig. 2 outside of $T$ and $T^{\prime}$ for some particular $u$ and $v$.

Now, we combine the two domains we have just introduced together.

Theorem 1 Let $\mathcal{D}_{1}$ and $\mathcal{D}_{2}$ be two Condorcet domains on disjoint sets of alternatives $A$ and $B$. Let $u \in \mathcal{D}_{1}$ and $v \in \mathcal{D}_{2}$ be arbitrary linear orders. Then,

$$
\left(\mathcal{D}_{1} \otimes_{1} \mathcal{D}_{2}\right)(u, v):=\left(\mathcal{D}_{1} \odot \mathcal{D}_{2}\right) \cup(u \oplus v)
$$

is a Condorcet domain. Moreover, if $\mathcal{D}_{1}$ and $\mathcal{D}_{2}$ are peak-pit domains, so is $\left(\mathcal{D}_{1} \otimes_{1} \mathcal{D}_{2}\right)(u, v)$.

Proof Let us fix $u$ and $v$ in this construction and denote $\left(\mathcal{D}_{1} \otimes_{1} \mathcal{D}_{2}\right)(u, v)$ as simply $\mathcal{D}_{1} \otimes_{1} \mathcal{D}_{2}$. If $a, b, c \in A$, then $\left(\mathcal{D}_{1} \otimes_{1} \mathcal{D}_{2}\right)_{\{a, b, c\}}=\left(\mathcal{D}_{1}\right)_{\{a, b, c\}}$, i.e. the restriction of $\mathcal{D}_{1} \otimes_{1} \mathcal{D}_{2}$ onto $\{a, b, c\}$ is the same as the restriction of $\mathcal{D}_{1}$. Hence, $\mathcal{D}_{1} \otimes_{1} \mathcal{D}_{2}$ satisfies the same never condition for $\{a, b, c\}$ as $\mathcal{D}_{1}$. For $x, y, z \in B$ the same thing happens. 
Suppose now $a, b \in A$ and $x \in B$. Then, $\left(\mathcal{D}_{1} \odot \mathcal{D}_{2}\right)_{\{a, b, x\}} \subseteq\{a b x, b a x\}$. Let us also assume (without loss of generality) that $u_{\{a, b\}}=\{a b\}$. Then, $(u \oplus v)_{\{a, b, x\}}=\{a b x, a x b, x a b\}$, hence

$$
\left(\mathcal{D}_{1} \otimes_{1} \mathcal{D}_{2}\right)_{\{a, b, x\}} \subseteq\{a b x, \text { bax }, a x b, x a b\},
$$

thus $\mathcal{D}_{1} \otimes_{1} \mathcal{D}_{2}$ satisfies $a N_{\{a, b, x\}} 3$. For $a \in A$ and $x, y \in B$ we have $\left(\mathcal{D}_{1} \odot \mathcal{D}_{2}\right)_{\{a, x, y\}} \subseteq\{a x y, a y x\}$. Let also $v_{\{x, y\}}=\{x y\} . \quad$ Then, $(u \oplus v)_{\{a, x, y\}}=\{a x y, x a y, x y a\}$, hence

$$
\left(\mathcal{D}_{1} \otimes_{1} \mathcal{D}_{2}\right)_{\{a, x, y\}} \subseteq\{\text { axy, ayx, xay, xya }\},
$$

thus $\mathcal{D}_{1} \otimes_{1} \mathcal{D}_{2}$ satisfies $y N_{\{a, x, y\}} 1$.

From the proof of Theorem 1, we can extract the following additional information.

Corollary 1 Let $\mathcal{D}_{1}$ and $\mathcal{D}_{2}$ be two Condorcet domains. Then,

(1) If $\mathcal{D}_{1}$ and $\mathcal{D}_{2}$ are ample, then $\left(\mathcal{D}_{1} \otimes_{1} \mathcal{D}_{2}\right)(u, v)$ is copious.

(2) If for any $a, b \in A$ and $x, y \in B$ with $a \succ_{u} b$ and $x \succ_{v} y$, then domain $\left(\mathcal{D}_{1} \otimes_{1}\right.$ $\left.\mathcal{D}_{2}\right)(u, v)$ satisfies $a N_{\{a, b, x\}} 3$ and $y N_{\{a, x, y\}} 1$.

Proof (1) The inequalities (4) and (5) become equalities if $\mathcal{D}_{1}$ and $\mathcal{D}_{2}$ are ample. (2) also follows from (4) and (5).

If $\mathcal{D}_{1}$ is a domain of maximal width on $[m]$ and $\mathcal{D}_{2}$ is a domain of maximal width on $[m+n] \backslash[m]$, then to obtain the original DKK-construction, we should choose $u=m(m-1) \cdots 1$ and $v=(m+n)(m+n-1) \cdots(m+1)$.

Proposition 2 If $|A|=m$ and $|B|=n$, then for any $u \in \mathcal{D}_{1}$ and $v \in \mathcal{D}_{2}$

$$
\left|\left(\mathcal{D}_{1} \otimes_{1} \mathcal{D}_{2}\right)(u, v)\right|=\left|\mathcal{D}_{1}\right|\left|\mathcal{D}_{2}\right|+\left(\begin{array}{c}
n+m \\
m
\end{array}\right)-1 .
$$

Proof We have $\left|\mathcal{D}_{1} \otimes_{1} \mathcal{D}_{2}\right|=\left|\mathcal{D}_{1}\right|\left|\mathcal{D}_{2}\right|$ and $|u \oplus v|=\left(\begin{array}{c}n+m \\ m\end{array}\right)$. These two sets have only one linear order in common which is $u v$. This proves (6).

Proposition 3 Let $\mathcal{D}_{1}$ and $\mathcal{D}_{2}$ be of maximal width with $u, \bar{u} \in \mathcal{D}_{1}$ and $v, \bar{v} \in \mathcal{D}_{2}$. Then, $\left(\mathcal{D}_{1} \otimes_{1} \mathcal{D}_{2}\right)(u, v)$ also has maximal width.

Proof We note that $\bar{u} \bar{v} \in \mathcal{D}_{1} \odot \mathcal{D}_{2}$. We also have $\overline{\bar{u}} \bar{v}=v u \in u \oplus v$, hence $\left(\mathcal{D}_{1} \otimes_{1}\right.$ $\left.\mathcal{D}_{2}\right)(u, v)$ has maximal width.

The following corollary will be important during the construction of large domains of maximal width.

Corollary 2 Let $\mathcal{D}_{1}$ be a domain of maximal width on $[m]$ with $e=1 \cdots m$ and $\bar{e}=m \cdots 1$ and $\mathcal{D}_{2}$ be a domain of maximal width on $[n] \backslash[m]$ with $f=$ 
$(m+1) \cdots n$ and $\bar{f}=n \cdots(m+1)$. Then, for the product $\left(\mathcal{D}_{1} \otimes_{1} \mathcal{D}_{2}\right)(u, v)$ to have maximal width with two completely reversed orders $1 \cdots n$ and $n \cdots 1$ we need to choose $u=\bar{e}$ and $v=\bar{f}$. In particular, if $\mathcal{D}_{1}$ and $\mathcal{D}_{2}$ are semi-connected, then so is $\left(\mathcal{D}_{1} \otimes_{1} \mathcal{D}_{2}\right)(\bar{e}, \bar{f})$.

Proof To prove this, we note that ef can be connected to $\bar{e} \bar{f}$ (which belongs both to $\mathcal{D}_{1} \odot \mathcal{D}_{2}$ and to $\left.\bar{e} \oplus \bar{f}\right)$ by a geodesic path within $\mathcal{D}_{1} \odot \mathcal{D}_{2}$ and $\bar{e} \bar{f}$, in turn, can be connected to $\bar{f} \bar{e}$ by a geodesic path within $\bar{e} \oplus \bar{f}$.

Theorem 2 Let $e \in\{f, g, h\}$ be one of the functions defined above. Then,

$$
e(n+m)>e(n) e(m) .
$$

Proof If $\mathcal{D}_{1}$ is one of the largest Condorcet domains on the set of $n$ alternatives of size $e(n)$ and $\mathcal{D}_{2}$ is the largest Condorcet domain on the set of $m$ alternatives of size $e(m)$, then, by Theorem $1,\left(\mathcal{D}_{1} \otimes_{1} \mathcal{D}_{2}\right)(u, v)$ is a Condorcet domain on the set of $n+m$ alternatives that has the size greater than $e(n) e(m)$ but smaller than or equal to $e(n+m)$. If both were peak-pit domains, then the product is also peak-pit and, moreover, by Proposition $3 u$ and $v$ could be chosen so that the product has maximum width if $\mathcal{D}_{1}$ and $\mathcal{D}_{2}$ had one. This proves the Eq. (7) for all three functions.

For function $f$, the Fishburn's replacement scheme allows to prove a slightly stronger inequality

$$
f(n+m-1) \geq f(n) f(m)
$$

(Lemma 1 in Fishburn (1996b)). This is because the replacement scheme used to obtain (8), given two Condorcet domains $\mathcal{D}_{1}$ and $\mathcal{D}_{2}$, produces a Condorcet domain for which, unlike our composition, some triples of alternatives satisfy never-middle condition.

If both $\mathcal{D}_{1}$ and $\mathcal{D}_{2}$ have maximal width, it is not true, however, that $\left(\mathcal{D}_{1} \otimes_{1}\right.$ $\left.\mathcal{D}_{2}\right)(u, v)$ will have maximal width for any $u \in \mathcal{D}_{1}$ and $v \in \mathcal{D}_{2}$. Let us take, for example, $\mathcal{D}_{1}=\{x=a b, \bar{x}=b a\}$ and $\mathcal{D}_{2}=\{u=c d e, v=d e c, w=d c e, \bar{u}=e d c\}$. Then, $\left(\mathcal{D}_{1} \otimes_{1} \mathcal{D}_{2}\right)(\bar{x}, \bar{u})$ has maximal width with abcde and edcba belonging to it, while $\left(\mathcal{D}_{1} \otimes_{1} \mathcal{D}_{2}\right)(\bar{x}, v)$ does not since $\bar{v} \notin \mathcal{D}_{2}$. In particular,

$$
\left(\mathcal{D}_{1} \otimes_{1} \mathcal{D}_{2}\right)(\bar{x}, \bar{u}) \neq\left(\mathcal{D}_{1} \otimes_{1} \mathcal{D}_{2}\right)(\bar{x}, v) .
$$

This indicates that the construction of the tensor product $\otimes_{1}$ may be useful in description and construction of Condorcet domains which do not satisfy the requirement of maximal width and we will see that in characterisation of Arrow's single-peaked domains.

Proposition 4 Let $\mathcal{D}_{1}$ and $\mathcal{D}_{2}$ be two connected Condorcet domains on disjoint sets of alternatives $A$ and $B$. Then, $\mathcal{D}=\left(\mathcal{D}_{1} \otimes_{1} \mathcal{D}_{2}\right)(u, v)$ is also connected. 
Proof Suppose that $\mathcal{D}_{1}$ and $\mathcal{D}_{2}$ are connected, and consider two neighbours $w, w^{\prime} \in$ $\mathcal{D}$ in $G_{\mathcal{D}}$. Due to connectedness of $\mathcal{D}_{1}$ and $\mathcal{D}_{2}$, if $w, w^{\prime} \in \mathcal{D}_{1} \odot \mathcal{D}_{2}$ they are connected by a series of swaps and since they are neighbours, they are connected by a single swap. If $w, w^{\prime} \in u \oplus v$ the situation is similar. Therefore, it is enough to consider the case when $w \in \mathcal{D}_{1} \odot \mathcal{D}_{2}$ and $w^{\prime} \in u \oplus v$. Then, $w$ and $w^{\prime}$ are connected by a series of swaps and any such path contains $u v$. Thus, $u v \in\left[w, w^{\prime}\right]_{\mathcal{D}}$. Hence, either $w=u v$ or $w^{\prime}=u v$ from which the statement follows.

Proposition 5 The following isomorphism holds:

$$
\left(F_{2}(a, b) \otimes_{1} F_{2}(c, d)\right)(b a, d c) \cong F_{4} .
$$

Proof We list orders of this domain as columns of the following matrix:

$$
\left[F_{2}(a, b) \odot F_{2}(c, d) \mid b a \oplus d c\right]=\left[\begin{array}{lllllllll}
a & a & b & b & b & b & d & d & d \\
b & b & a & a & d & d & b & b & c \\
c & d & c & d & a & c & c & a & b \\
d & c & d & c & c & a & a & c & a
\end{array}\right] .
$$

We see that the following never conditions are satisfied: $b N_{\{a, b, c\}} 3, b N_{\{a, b, d\}} 3$, $c N_{\{a, c, d\}} 1, c N_{\{b, c, d\}} 1$. Hence, the mapping $1 \rightarrow a, 2 \rightarrow b, 3 \rightarrow c$ and $4 \rightarrow d$ is an isomorphism of $F_{4}$ onto the tensor product $\left(F_{2}(a, b) \otimes_{1} F_{2}(c, d)\right)(b a, d c)$.

The isomorphism (9) is very nice but unfortunately, as we will see in example that follows, for larger $m, n$ we have no such isomorphisms. Moreover, it appears that for two maximal Condorcet domains $\mathcal{D}_{1}$ and $\mathcal{D}_{2}$ on sets $A$ and $B$, respectively, $\left(\mathcal{D}_{1} \otimes_{1} \mathcal{D}_{2}\right)(u, v)$ may not be maximal on $A \cup B$. Here is an example.

Example 1 Let us calculate $\mathcal{E}:=F_{3}(1,2,3) \otimes_{1} F_{2}(4,5)(321,54)$ :

$$
\left[\begin{array}{lllllllllllllllll}
1 & 2 & 2 & 3 & 1 & 2 & 2 & 3 & 3 & 3 & 5 & 3 & 3 & 3 & 5 & 5 & 5 \\
2 & 1 & 3 & 2 & 2 & 1 & 3 & 2 & 2 & 5 & 3 & 2 & 5 & 5 & 3 & 3 & 4 \\
3 & 3 & 1 & 1 & 3 & 3 & 1 & 1 & 5 & 2 & 2 & 5 & 2 & 4 & 2 & 4 & 3 \\
4 & 4 & 4 & 4 & 5 & 5 & 5 & 5 & 1 & 1 & 1 & 4 & 4 & 2 & 4 & 2 & 2 \\
5 & 5 & 5 & 5 & 4 & 4 & 4 & 4 & 4 & 4 & 4 & 1 & 1 & 1 & 1 & 1 & 1
\end{array}\right] .
$$

There are 17 linear orders in this domain. It is known, however, that $F_{5}$ has 20 (Fishburn 1996a) but this fact alone does not mean non-maximality of $\mathcal{E}$. By Corollary 1(1) this domain is copious. By its construction (see Corollary 1(2)), it satisfies just three inversion triples:

$$
[1,4,5], \quad[2,4,5], \quad[3,4,5] .
$$

Now, we see that there are two more linear orders 23514 and 23541 that satisfy these conditions. Hence, $\mathcal{E}$ is not maximal. In particular, $\mathcal{E}$ is not isomorphic to $F_{5}$. Therefore, the isomorphism (9) is just one of a kind. 
Despite not providing maximal Condorcet domains, DKK-construction allowed (Danilov and Karzanov 2012) to refute a long standing conjecture (Fishburn 1996a; Galambos and Reiner 2008) that $g(n)=\left|F_{n}\right|$, where $F_{n}$ is the $n$th Fishburn's domain. In other words, they showed that for large $n$, it is not true that $F_{n}$ is the largest peak-pit Condorcet domain of maximal width on $n$ alternatives.

Below by $F_{n} \otimes_{1} F_{m}$, we will mean the product

$$
\left(\mathcal{D}_{1} \otimes_{1} \mathcal{D}_{2}\right)(\bar{e}, \bar{f})
$$

where $\mathcal{D}_{1}$ is the $n$th Fishburn's domain on the set of alternatives $[n], \mathcal{D}_{2}$ is the $m$ th Fishburn's domain on the set of alternatives $[n+m] \backslash[n]$, and $e=12 \cdots n$, $f=(n+1)(n+2) \cdots(n+m)$. (As we previously noted, such a choice of $u$ and $v$ in $\left(\mathcal{D}_{1} \otimes_{1} \mathcal{D}_{2}\right)(u, v)$ secures the maximal width of the product.)

To disprove the aforementioned conjecture, Danilov and Karzanov (2012) showed that $\left|F_{21} \otimes_{1} F_{21}\right|>\left|F_{42}\right|$ which implies that $g(42)>\left|F_{42}\right|$. Our calculations, using exact formulas (3) and (6) show that

$$
\left|F_{n} \otimes_{1} F_{n}\right|<\left|F_{2 n}\right|
$$

for $2<n \leq 19$ but $4611858343415=\left|F_{20} \otimes_{1} F_{20}\right|>\left|F_{40}\right|=4549082342996$ which shows that $g(40)>\left|F_{40}\right|$. Later in the paper, we will further improve this inequality.

The fact that the Fishburn's hypothesis is not true also follows from the unpublished result of Ondrej Bilka announced in Felsner and Valtr (2011). It states that for large $n$, it is true that $g(n)>2.0767^{n}$. Since this lower bound asymptotically grows faster than the cardinality of Fishburn's domain (3), the result follows. Below, we give a proof of Bilka's bound.

Another use of DKK-construction, due to Proposition 2, allows us to note, for example, that $g(x+y)>g(x) g(y)$ and $g(a x)>g(x)^{a}$ for any positive integer $a$, and a similar inequalities hold for function $h$. This helps us to find lower bounds for functions $g, h$ with the help of the following theorem that is analogous (but slightly weaker) than Fishburn's Lemma 2 in Fishburn (1996b) which was proved for function $f$ and was based on the replacement scheme. We remind the reader the statement of Fishburn's theorem.

Theorem 3 (Fishburn 1996b) For any natural $k$, positive $\epsilon$, and for sufficiently large $n$, we have

$$
f(n)>\left(f(k)^{\frac{1}{k-1}-\epsilon}\right)^{n}
$$

For two other functions, we can now prove a similar theorem.

Theorem 4 For any natural $k$, positive $\epsilon$, and for sufficiently large $n$, we have

$$
e(n)>\left(e(k)^{\frac{1}{k}-\epsilon}\right)^{n}
$$

where $e \in\{g, h\}$. 
Proof We note that by Theorem 2 for any positive integer $a$, we have $e(a x)>e(x)^{a}$. Let us divide $n$ by $k$ with remainder: $n=a k+b$, where $0 \leq b<k$. Then, we will have

$$
e(n)>(e(k))^{a} e(b)=(e(k))^{(n-b) / k} e(b) \geq(e(k))^{(n-b) / k} \geq c(k)(e(k))^{n / k},
$$

where $c(k)>0$ is a coefficient, which does not depend on $n$. For any positive $\epsilon$ and sufficiently big $n$, we have the result.

With the help of Theorem 4, we can now get a lower bound for $g$.

Theorem $5 \quad g(n)>2.0767^{n}$ for all large $n$.

Proof Using Proposition 2, we can calculate the size of domain $\mathcal{D}=\left(F_{27} \otimes_{1}\right.$ $\left.F_{27}\right) \otimes_{1}\left(F_{27} \otimes_{1} F_{27}\right)$ which is a connected Condorcet domain of maximal width on 108 alternatives with $|\mathcal{D}|=1.8917 \cdot 10^{34}$ linear orders. We note that $|\mathcal{D}|^{1 / 108}=2.07672 \ldots$. We can choose a small $\epsilon$ such that $|\mathcal{D}|^{1 / 108-\epsilon}=2.0767$. Then, from Theorem 4, we get the result:

$$
g(n)>\left(g(k)^{1 / k-\epsilon}\right)^{n}>\left(|\mathcal{D}|^{1 / k-\epsilon}\right)^{n}=2.0767^{n} .
$$

This confirms the result announced in Felsner and Valtr (2011).

\section{A new construction}

As we have seen in Example 1, the DKK-construction does not guarantee the maximality of the product of two maximal domains, and we could add two more linear orders without violating all never conditions. Let us extend their construction. We need to introduce a necessary notation first.

Given a linear order $x=x_{1} \cdots x_{n}$ in $\mathcal{L}(A)$, we can view it as a concatenation $x=x^{1} x^{2}$ of two suborders, where for some $s \in\{0,1, \ldots, n\}$, we have $x^{1}=x_{1} \ldots x_{s}$ the top part of $x$ and by $x^{2}=x_{s+1} \cdots x_{n}$ the bottom part of it. We allow for a trivial splitting when $s=0$ and $x^{1}$ is empty or $s=n$ and $x^{2}$ is empty.

Given a domain of linear orders $\mathcal{D} \subseteq \mathcal{L}(A)$ and a linear order $x=x_{i_{1}} \ldots x_{i_{k}}$ defined on a subset $X=\left\{x_{i_{1}} \ldots x_{i_{k}}\right\} \subseteq A$, we define the upper and lower contour sets of $x$ as

$$
\begin{aligned}
& U_{\mathcal{D}}(x)=\{z \in \mathcal{L}(A \backslash X) \mid z x \in \mathcal{D}\}, \\
& L_{\mathcal{D}}(x)=\{y \in \mathcal{L}(A \backslash X) \mid x y \in \mathcal{D}\} .
\end{aligned}
$$

These sets can be empty sometimes. In addition, if $x$ is empty, $U_{\mathcal{D}}(x)=L_{\mathcal{D}}(x)=\mathcal{D}$.

For example, if $\mathcal{D}=F_{4}$, given in (2), then $U_{\mathcal{D}}(34)=\{12,21\}$ and $L_{\mathcal{D}}(12)=\{34,43\}$.

Let $\mathcal{D}_{1}$ and $\mathcal{D}_{2}$ be two Condorcet domains on disjoint sets of alternatives $A$ and $B$. Let $u \in \mathcal{D}_{1}$ and $v \in \mathcal{D}_{2}$ be arbitrary linear orders. Let also $u=u^{1} u^{2}$ and $v=v^{1} v^{2}$ be any splittings of $u$ and $v$. Let us define the domain 


$$
u \boxplus v:=\bigcup\left(U_{\mathcal{D}_{1}}\left(u^{2}\right) \odot\left(u^{2} \oplus v^{1}\right) \odot L_{\mathcal{D}_{2}}\left(v^{1}\right)\right),
$$

where the union is over all splittings of $u$ and $v$ (including the trivial ones).

Proposition $6 \quad u \boxplus v \supseteq u \oplus v$.

Proof Under the trivial splittings of $u$ and $v$, we have $v^{1}=v, u^{2}=u$ and $L_{\mathcal{D}_{2}}\left(v^{1}\right)=$ $\left(U_{\mathcal{D}_{1}}\left(u^{2}\right)=\emptyset\right.$ so $U_{\mathcal{D}_{1}}\left(u^{2}\right) \odot\left(u^{2} \oplus v^{1}\right) \odot L_{\mathcal{D}_{2}}\left(v^{1}\right)=u \oplus v$.

Lemma 1 Let $\mathcal{D}_{1}$ and $\mathcal{D}_{2}$ be two Condorcet domains on disjoint sets of alternatives $A$ and $B$. Let $u \in \mathcal{D}_{1}$ and $v \in \mathcal{D}_{2}$ be arbitrary linear orders. Then, the domain $u \boxplus v$ satisfies all the never conditions that $\left(\mathcal{D}_{1} \otimes_{1} \mathcal{D}_{2}\right)(u, v)$ satisfies.

Proof Let us consider an arbitrary element $w \in U_{\mathcal{D}_{1}}\left(u^{2}\right) \odot\left(u^{2} \oplus v^{1}\right) \odot L_{\mathcal{D}_{2}}\left(v^{1}\right)$ for $u=u^{1} u^{2} \in \mathcal{D}_{1}$ and $v=v^{1} v^{2} \in \mathcal{D}_{2}$, where $u^{1} \in \mathcal{L}\left(A_{1}\right)$ and $u^{2} \in \mathcal{L}\left(A_{2}\right)$ for a partition $A=A_{1} \cup A_{2}$ and where $v^{1} \in \mathcal{L}\left(B_{1}\right)$ and $v^{2} \in \mathcal{L}\left(B_{2}\right)$ for a partition $B=B_{1} \cup B_{2}$. Suppose $w=\tilde{u} \odot\left(u^{2} \oplus v^{1}\right) \odot \tilde{v}$, where $\tilde{u} \in U_{\mathcal{D}_{1}}\left(u^{2}\right)$ and $\tilde{v} \in L_{\mathcal{D}_{2}}\left(v_{1}\right)$. We note that, by the definition, $\tilde{u} u^{2} \in \mathcal{D}_{1}$ and $v^{1} \tilde{v} \in \mathcal{D}_{2}$. Hence, for any triple $\{a, b, c\} \subseteq A$ or $\{x, y, z\} \subseteq B$, the order $w$ will satisfy the same never condition as in $\mathcal{D}_{1}$ and $\mathcal{D}_{2}$, respectively.

Suppose, for example, that $a \in A_{2}, x, y \in B$ with $x \succ_{v} y$. Then, by Corollary 1(2) $\left(\mathcal{D}_{1} \otimes_{1} \mathcal{D}_{2}\right)(u, v)$ satisfies $y N_{\{a, x, y\}} 1$. It is easy to see that $w$ satisfies the same never condition as $y$ cannot come ahead of both $a$ and $x$.

Example 2 As we saw in Example 1, the DKK-construction does not capture two linear orders 23514 and 23541 which results in the product being not maximal. However, if we define $u^{1}=32, \quad u^{2}=1, \quad v^{1}=54$ and $v^{2}=\emptyset$, then $U_{\mathcal{D}_{1}}\left(u^{2}\right)=U_{\mathcal{D}_{1}}(1)=\{23,32\}, \quad L_{\mathcal{D}_{2}}\left(v^{1}\right)=\emptyset, \quad u^{2} \oplus v^{1}=1 \oplus 54=\{154,514,541\}$ and thus 23514 and 23541 will be then captured by

$$
U_{\mathcal{D}_{1}}\left(u_{2}\right) \odot\left(u^{2} \oplus v^{1}\right) \odot L_{\mathcal{D}_{2}}\left(v^{1}\right) \in u \boxplus v .
$$

Now, given two Condorcet domains $\mathcal{D}_{1}$ and $\mathcal{D}_{2}$, we define a new domain

$$
\left(\mathcal{D}_{1} \otimes_{2} \mathcal{D}_{2}\right)(u, v):=\left(\mathcal{D}_{1} \odot \mathcal{D}_{2}\right) \cup(u \boxplus v) .
$$

By Corollary 1 and Lemma 1 , it is a Condorcet domain which is copious if $\mathcal{D}_{1}$ and $\mathcal{D}_{2}$ are ample and peak-pit domain if $\mathcal{D}_{1}$ and $\mathcal{D}_{2}$ were peak-pit.

We saw in Example 2 that the new construction-concatenation + shuffle scheme-yields a domain which is strictly bigger than the DKK-construction does. Moreover, the concatenation + shuffle scheme always yields a maximal Condorcet domain which will be proved in the following theorem.

Theorem 6 For any two ample maximal Condorcet domains $\mathcal{D}_{1}$ and $\mathcal{D}_{2}$ and any $u \in \mathcal{D}_{1}, v \in \mathcal{D}_{2}$, the domain 


$$
\mathcal{D}=\left(\mathcal{D}_{1} \otimes_{2} \mathcal{D}_{2}\right)(u, v)
$$

is also a maximal Condorcet domain.

Proof Suppose that $\mathcal{D}$ is not a maximal Condorcet domain, then there is a linear order $w$, not belonging to this set, which addition, however, does not violate all the never conditions that $\left(\mathcal{D}_{1} \otimes_{2} \mathcal{D}_{2}\right)(u, v)$ satisfies. Let $w_{\mathcal{D}_{1}}=\tilde{u}$ and $w_{\mathcal{D}_{2}}=\tilde{v}$. Since domains $\mathcal{D}_{1}$ and $\mathcal{D}_{2}$ are maximal, these restrictions belong to $\mathcal{D}_{1}$ and $\mathcal{D}_{2}$, respectively, and we have $w \in \tilde{u} \oplus \tilde{v}$. If $w=\tilde{u} \tilde{v}$, then $w \in \mathcal{D}_{1} \odot \mathcal{D}_{2}$. Thus, we may assume that $w \neq \tilde{u} \tilde{v}$.

If there are splittings $\tilde{u}=\tilde{u}^{1} \tilde{u}^{2}$ and $\tilde{v}=\tilde{v}^{1} \tilde{v}^{2}, u=u^{1} u^{2}$, and $v=v^{1} v^{2}$ such that $\tilde{u}^{2}=u^{2}$ and $\tilde{v}^{1}=v^{1}$, then $w \in U_{\mathcal{D}_{1}}\left(u^{2}\right) \odot\left(u^{2} \oplus v^{1}\right) \odot L_{\mathcal{D}_{2}}\left(v^{1}\right)$ and hence $w \in \mathcal{D}$. Thus, we may assume that such splittings do not exist. In particular, we may assume that either the minimal elements of $\tilde{u}^{2}$ and $u^{2}$ are different or the maximal elements of $\tilde{v}^{1}$ and $v^{1}$ are different.

Suppose that the minimal elements $a$ and $b$ in linear orders $\tilde{u}, u$, respectively, are different and suppose without loss of generality that $a \succ_{u} b$. Let $x$ be the maximal element in linear order $\tilde{v}=v$. Then, for the triple of alternatives $\{a, b, x\}$, by Corollary 1 the condition $a N_{\{a, b, x\}} 3$ is satisfied and also $\mathcal{D}_{\{a, b, x\}}=$ $\{a b x, b a x, a x b, x a b\}$ as by Corollary $1 \mathcal{D}$ is copious. However, since $w \neq \tilde{u} \tilde{v}$, we will have $x \succ_{w} a$ which implies that either $b x a$ or $x b a$ is in $\mathcal{D}_{\{a, b, x\}}$, a contradiction. The case when the maximal elements $x$ and $y$ in linear orders $\tilde{v}, v$, respectively, are different is similar.

The new definition enables recursive construction of domains.

Theorem 7 Let $\mathcal{D}_{1}$ be an Arrow's single-peaked domain on the set of alternatives $A$ and $\mathcal{D}_{2}$ be a trivial domain on a one-element set $\{b\}$, where $b \notin A$. Let $u \in \mathcal{D}_{1}$ and $v=b \in \mathcal{D}_{2}$. Then, $\mathcal{D}=\left(\mathcal{D}_{1} \otimes_{2} \mathcal{D}_{2}\right)(u, v)$ is also Arrow's single-peaked. If $\mathcal{D}_{1}$ were maximal, then $\mathcal{D}$ would be also maximal.

Proof By Corollary 1, we add to the set of never conditions governing $\mathcal{D}_{1}$ the set

$$
\left\{a N_{\left\{a, a^{\prime}, b\right\}} 3, \quad a, a^{\prime} \in A, \quad a \succ_{u} a^{\prime}\right\},
$$

hence the obtained domain $\mathcal{D}$ is also defined by the never-bottom conditions, hence, it is Arrow's single-peaked. If $\mathcal{D}_{1}$ were maximal, then $\mathcal{D}$ would be also maximal by Theorem 6.

An alternative of an Arrow's single-peaked domain $\mathcal{D}$ is called terminal if it is a bottom alternative of at least one linear order in $\mathcal{D}$. In each maximal Arrow's singlepeaked domain, there are exactly two terminal alternatives. A pair of orders in Arrow's single-peaked domain is called extremal if top and bottom alternatives are reversed in these orders. Each Arrow's single-peaked domain has exactly one pair of extremal linear orders (Slinko 2019).

Lemma 2 If two maximal Arrow's single-peaked domains on the set of $n$ alternatives have identical extremal orders with top/bottom alternatives $a, b$ and 
these domains have identical restrictions on the set $A \backslash\{a, b\}$, then these domains are identical.

Proof Let us prove by induction. The statement is true for $n=3$.

Let us consider two maximal Arrow's single-peaked domains $\mathcal{D}_{1}$ and $\mathcal{D}_{2}$ on the set $A$ of $n$ alternatives with the same extremal orders $e_{1}, e_{2}$, which have the following structure $e_{1}=a v_{1} b, e_{2}=b v_{2} a$ so $a$ and $b$ are terminal. Both domains have the same restrictions on the set $A \backslash\{a, b\}$ which is domain $\mathcal{D}_{3}$. Let us define the following domains $\quad \mathcal{D}_{1 a}=U_{\mathcal{D}_{1}}(a), \quad \mathcal{D}_{1 b}=U_{\mathcal{D}_{1}}(b), \quad \mathcal{D}_{2 a}=U_{\mathcal{D}_{2}}(a)$, $\mathcal{D}_{2 b}=U_{\mathcal{D}_{2}}(b)$. These domains are restrictions of respective domains $\mathcal{D}_{1}$ and $\mathcal{D}_{2}$ onto the sets $A \backslash\{a\}$ and $A \backslash\{b\}$. According to Lemma 4.3 in Slinko (2019), all these are maximal Arrow's single-peaked domains on the respective sets of $n-1$ alternatives.

Let us consider domain $\mathcal{D}_{1 b}$. We claim that $a$ is a terminal alternative in this domain and $e_{1 b}=a v_{1}$ is one of its extremal orders. Assume for a moment that $a$ is not terminal and $x$ and $y$ are terminal for $\mathcal{D}_{1 b}$ instead. Then, for the triple $\{a, x, y\}$ in $\mathcal{D}$, no never-bottom condition is satisfied. Another extremal linear order starts from the last alternative in order $v_{1}$, let us call it $c$, and has structure $e_{2 b}=c v_{3} a$, where $c v_{3} \in \mathcal{D}_{3}$. All these arguments are applicable also to $\mathcal{D}_{2 b}$ so their extremal orders coincide. Thus, the two domains $\mathcal{D}_{1 b}$ and $\mathcal{D}_{2 b}$ are maximal Arrow's single-peaked, have identical extremal linear orders, and have identical restrictions on the set $A \backslash\{a, b, c\}$. By the induction hypothesis, we have $\mathcal{D}_{1 b}=\mathcal{D}_{2 b}$. Similarly, we have $\mathcal{D}_{1 a}=\mathcal{D}_{2 a}$. This implies $\mathcal{D}_{1}=\mathcal{D}_{2}$.

Theorem 8 Each Arrow's single-peaked domain $\mathcal{D}$ on the set A of $n$ alternatives is a composition $\left(\mathcal{D}_{1} \otimes_{2} \mathcal{D}_{2}\right)(u, v)$ of an Arrow's single-peaked domain $\mathcal{D}_{1}$ on the set of $n-1$ alternatives $A \backslash\{a\}$ and the trivial domain $\mathcal{D}_{2}$ on $\{a\}$, where $u \in \mathcal{D}_{1}$ and $v=a$.

Proof Let us consider extremal orders $e_{1}, e_{2}$ of $\mathcal{D}$ which are $e_{1}=a w_{1} b, e_{2}=b w_{2} a$ for terminal alternatives $a$ and $b$ and some orders $w_{1}, w_{2} \in \mathcal{L}(A \backslash\{a, b\})$.

Let $\mathcal{D}_{1}=U_{\mathcal{D}}(a), \mathcal{D}_{2}=\{a\}, u=w_{1} b$ and $v=a$, then by Theorem 7 , domain $\mathcal{E}=\left(\mathcal{D}_{1} \otimes_{2} \mathcal{D}_{2}\right)(u, v)$ is a maximal Arrow's single-peaked domain. In addition, we have $e_{2}=b w_{2} a \in \mathcal{D}_{1} \odot \mathcal{D}_{2} \subseteq \mathcal{E}$.

As in the proof of Theorem $7, b$ is a terminal alternative of $\mathcal{D}_{1}$. Let us consider $\mathcal{D}_{3}=U_{\mathcal{D}_{1}}(b)$. Again by Theorem 7, it is a maximal Arrow's single-peaked domain on $A \backslash\{a, b\}$. Due to the maximality of the latter $w_{1} \in \mathcal{D}_{3}$, hence $u=w_{1} b \in \mathcal{D}_{1}$. Now, $e_{1}=a w_{1} b \in u \oplus a \subseteq u \boxplus v \subseteq \mathcal{E}$.

Thus, $\mathcal{E}$ contains the same extremal orders $e_{1}$ and $e_{2}$, and has the same restriction on the set $A \backslash\{a, b\}$ as domain $\mathcal{D}$. From Lemma $2, \mathcal{D}=\mathcal{E}$.

Corollary 3 Each Arrow's single-peaked domain can be obtained by iterated application of concatenation + shuffle scheme to the trivial domain.

One additional case is noteworthy. We remind the reader that a domain of linear orders is called (Black's) single-peaked if there exists a left-to-right arrangement (axis) of alternatives such that an upper contour set of any linear order in this 
domain is convex subset of the axis (Black 1948). Up to an isomorphism, we can assume that the axis is $12 \cdots n$.

Corollary 4 Let $\mathcal{D}_{1}$ be Black's single-peaked domain on the set of alternatives $[n]$ with the axis $12 \ldots n$ and $\mathcal{D}_{2}$ be the trivial domain on a one-element set $\{n+1\}$. Let $u=n(n-1) \ldots 21$ and $v=n+1$. Then, $\left(\mathcal{D}_{1} \otimes_{2} \mathcal{D}_{2}\right)(u, v)$ is Black's singlepeaked domain with axis $12 \ldots(n+1)$.

Zhan (2019) observed this recursive property of the single-peaked domains considering their tilling representations.

Not all peak-pit domains are reducible in the sense of Corollary 3. Up to an isomorphism, there are 18 peak-pit domains of maximal width with five alternatives (Li et al. 2021). Ten of them can be obtained from smaller domains by the concatenation + shuffle scheme.

\section{Constructing large peak-pit Condorcet domains}

We again take two Fishburn's domains $F_{n}$ and $\bar{F}_{n}$ (which are not isomorphic but flip-isomorphic) as building blocks for constructing new large domains. However, now we use the new construction to combine them. First, we will look at the highest possible value $T_{n}$ of the cardinality of the product

$$
\left(\mathcal{D} \otimes_{2} \mathcal{E}\right)(u, v),
$$

where $\mathcal{D} \in\left\{F_{\left\lfloor\frac{n}{2}\right\rfloor}, \bar{F}_{\left\lfloor\frac{n}{2}\right\rfloor}\right\}$ and $\mathcal{E} \in\left\{F_{\left\lceil\frac{n}{2}\right\rceil}, \bar{F}_{\left\lceil\frac{n}{2}\right\rceil}\right\}$, with arbitrary $u \in \mathcal{D}$ and $v \in \mathcal{E}$.

Second, if we restrict ourselves with domains of maximal width, we have to choose $u$ and $v$ as shown in Corollary 2. The highest possible value of the cardinality of the product under these conditions will be denoted as $S_{n}$. As we will see, Table 1 shows the importance of choosing linear orders $u$ and $v$ for

Table 1 Products of Fishburn's domains under $\otimes_{1}$, under $\otimes_{2}$ with $u$ and $v$ chosen to secure maximal width, and $\otimes_{2}$ where $u$ and $v$ are chosen to get the size as large as possible

\begin{tabular}{lllll}
\hline$n$ & $\left|F_{n}\right|$ & $\left|F_{\left\lfloor\frac{n}{2}\right\rfloor} \otimes_{1} F_{\left\lceil\frac{n}{2}\right\rceil}\right|$ & $S_{n}$ & $T_{n}$ \\
\hline 2 & 2 & 2 & 2 & 2 \\
3 & 4 & 4 & 4 & 4 \\
4 & 9 & 9 & 9 & 9 \\
5 & 20 & 17 & 19 & 19 \\
6 & 45 & 35 & 42 & 42 \\
7 & 100 & 70 & 85 & 93 \\
8 & 222 & 150 & 183 & 211 \\
9 & 488 & 305 & 392 & 462 \\
10 & 1069 & 651 & 860 & 1028 \\
11 & 2324 & 1361 & 1791 & 2233 \\
12 & 5034 & 2948 & 3843 & 4916 \\
13 & 10840 & 6215 & 8214 & 10668 \\
14 & 23266 & 13431 & 17828 & 23360 \\
\hline
\end{tabular}


concatenation + shuffle scheme since the resulting domains may have different cardinality depending on the selected orders. This is why $S_{n}$ is generally smaller than $T_{n}$. Our goal is to see for which $n$ we have $S_{n}>\left|F_{n}\right|$ and for which $n$ it is true that $T_{n}>\left|F_{n}\right|$. In the following table, we present the results of our calculations.

We see that for neither $S_{n}$, nor $T_{n}$ exceeds $\left|F_{n}\right|$ for $n<14$. But for $n=14$

$$
T_{14}=23360>23266=\left|F_{14}\right|,
$$

which means that $h(14)>\left|F_{14}\right|$. However, as we will see later, we can do even better.

When we restrict ourselves with Condorcet domains of maximal width (the classical case), we have much less freedom. As a result $S_{n}$ reaches $\left|F_{n}\right|$ much later. We have $S_{n}<\left|F_{n}\right|$ for $n=5, \ldots, 33$ but

$$
S_{34}=\left|\left(F_{17} \otimes_{2} F_{17}\right)(\bar{e}, \bar{f})\right|=60034795696>\left|F_{34}\right|=59921782301 .
$$

This gives us

Theorem $9 \quad g(34)>\left|F_{34}\right|$.

This significantly improves the result $g(42)>\left|F_{42}\right|$ proved by Danilov and Karzanov (2012).

For the general case, the choice of linear orders $u$ and $v$ for concatenation + shuffle scheme influences the structure of resulting domains even when they have equal cardinality. Let us consider the following example.

Example 3 Let us find $\mathcal{E}_{1}:=\left(F_{3} \otimes_{2} F_{2}\right)(321,54)$ :

$$
\left[\begin{array}{lllllllllllllllllll}
1 & 1 & 2 & 2 & 2 & 2 & 2 & 2 & \mathbf{3} & \mathbf{3} & \mathbf{3} & \mathbf{3} & \mathbf{3} & \mathbf{3} & \mathbf{3} & 5 & 5 & 5 & 5 \\
2 & 2 & 1 & 1 & 3 & 3 & 3 & 3 & \mathbf{2} & \mathbf{2} & \mathbf{2} & \mathbf{2} & 5 & 5 & 5 & 3 & 3 & 3 & 4 \\
3 & 3 & 3 & 3 & 1 & 1 & 5 & 5 & \mathbf{1} & \mathbf{1} & 5 & 5 & 2 & 2 & 4 & 2 & 2 & 4 & 3 \\
4 & 5 & 4 & 5 & 4 & 5 & 1 & 4 & 4 & \mathbf{5} & 1 & 4 & 1 & 4 & 2 & 1 & 4 & 2 & 2 \\
5 & 4 & 5 & 4 & 5 & 4 & 4 & 1 & 5 & \mathbf{4} & 4 & 1 & 4 & 1 & 1 & 4 & 1 & 1 & 1
\end{array}\right]
$$

and $\mathcal{E}_{2}:=\left(F_{3} \otimes_{2} F_{2}\right)(213,45)$ :

$$
\left[\begin{array}{lllllllllllllllllll}
1 & 1 & 1 & 1 & \mathbf{2} & \mathbf{2} & \mathbf{2} & \mathbf{2} & \mathbf{2} & \mathbf{2} & \mathbf{2} & \mathbf{2} & \mathbf{2} & 3 & 3 & 4 & 4 & 4 & 4 \\
2 & 2 & 2 & 2 & \mathbf{1} & \mathbf{1} & \mathbf{1} & \mathbf{1} & 3 & 3 & 4 & 4 & 4 & 2 & 2 & 2 & 2 & 2 & 5 \\
3 & 3 & 4 & 4 & \mathbf{3} & \mathbf{3} & 4 & 4 & 1 & 1 & 1 & 1 & 5 & 1 & 1 & 1 & 1 & 5 & 2 \\
4 & 5 & 3 & 5 & \mathbf{4} & 5 & 3 & 5 & 4 & 5 & 3 & 5 & 1 & 4 & 5 & 3 & 5 & 1 & 1 \\
5 & 4 & 5 & 3 & \mathbf{5} & 4 & 5 & 3 & 5 & 4 & 5 & 3 & 3 & 5 & 4 & 5 & 3 & 3 & 3
\end{array}\right] .
$$

Both domains have equal cardinality, but they are neither isomorphic, nor flipisomorphic. Domain $\mathcal{E}_{2}$ has alternative 2 with the lower contour set of cardinality 9 while the highest possible cardinality of a lower contour set of domain $\mathcal{E}_{1}$ is 7 (of alternative 3).

To explain why $\left(\mathcal{D}_{1} \otimes_{2} \mathcal{D}_{2}\right)(u, v)$ may have different cardinalities depending on $u$ and $v$, let us consider $F_{3}=\{123,213,231,321\}$. In $F_{3}$, alternative 1 has the lower 
contour set of cardinality 1 , while alternative 2 has its lower contour set of cardinality 2 . Hence, the domain $\left(\mathcal{D} \otimes_{2} F_{3}\right)(u, v)$ with $v=213$ has higher cardinality of $\left(u \oplus v^{1}\right) \odot L_{F_{3}}\left(v^{1}\right)$ for $v^{1}=2$ than such domain with $v=123$ and $v^{1}=1$.

Given domains $\mathcal{D}_{1}$ and $\mathcal{D}_{2}$ on sets of alternatives $A$ and $B$, respectively, for generating a large concatenation + shuffle domain $\left(\mathcal{D}_{1} \otimes_{2} \mathcal{D}_{2}\right)(u, v)$ with large contour sets (for further use in the concatenation + shuffle scheme), we use the following heuristic greedy algorithm to find the 'best' orders $u$ and $v$.

Algorithm 1 (Heuristically selecting $u$ and $v$ in $\oplus_{2}$ ) Let $\mathcal{D}_{1}$ and $\mathcal{D}_{2}$ be two Condorcet domains on sets of alternatives $A$ and $B$ of cardinalities $m$ and $n$, respectively. Firstly, to find $v \in \mathcal{D}_{2}$ we find an alternative $v_{1} \in B$ with the largest $L_{\mathcal{D}_{2}}\left(v_{1}\right)$, (if two alternatives have the same number of first placements, we take any of them), then we find an alternative $v_{2} \in B$ with the largest $L_{\mathcal{D}_{2}}\left(v_{1} v_{2}\right)$, etc. Then we set $v=v_{1} \ldots v_{n}$. To find $u \in \mathcal{D}_{1}$ with upper contour sets of the highest cardinality we find alternative $u_{m} \in A$, with the largest $U_{\mathcal{D}_{1}}\left(u_{m}\right)$, then we find an alternative $u_{m-1} \in A$ with the largest $U_{\mathcal{D}_{1}}\left(u_{m-1} u_{m}\right)$, etc. Then we set $u=u_{1} \ldots u_{m}$.

When using this heuristics for choosing $u$ and $v$ in the product $\left(F_{m} \otimes_{2} F_{n}\right)(u, v)$, we get a domain that have usually larger lower and upper contour sets than Fishburn's domain $F_{m+n}$. Due to this, we obtain

$$
\max _{u, v}\left|\left(\left(F_{4} \otimes_{2} F_{3}\right) \otimes_{2}\left(F_{4} \otimes_{2} F_{3}\right)\right)(u, v)\right| \geq 24481>23360=\max _{u, v}\left|\left(F_{7} \otimes_{2} F_{7}\right)(u, v)\right|
$$

despite $\max _{u, v}\left|\left(F_{4} \otimes_{2} F_{3}\right)(u, v)\right|<\left|F_{7}\right|$. We also discovered that

$$
\max _{u, v}\left|\left(\left(F_{4} \otimes_{2} F_{3}\right) \otimes_{2}\left(F_{3} \otimes_{2} F_{3}\right)\right)(u, v)\right| \geq 10940>10840=\left|F_{13}\right|,
$$

which proves one of the main results of this paper.

Theorem $10 h(13)>\left|F_{13}\right|$.

The cardinality and the structure of the biggest domains that we found are summarised in Table 2 in which for better readability we removed the subscript from $\otimes_{2}$ and did not mention the orders $u, v$ used in the construction. These orders are found using Algorithm 1.

Having $F_{4}=F_{2} \otimes_{2} F_{2}, F_{3}=F_{2} \otimes_{2} F_{1}$ and $F_{2}=F_{1} \otimes_{2} F_{1}$, we note that all presented domains are constructed via iterative mixing of trivial domains. For example, we have

$$
\begin{array}{r}
\left(F_{3} \otimes F_{3}\right) \otimes\left(F_{3} \otimes F_{2}\right)=\left(\left(F_{2} \otimes F_{1}\right) \otimes\left(F_{2} \otimes F_{1}\right)\right) \otimes\left(\left(F_{2} \otimes F_{1}\right) \otimes\left(F_{1} \otimes F_{1}\right)\right)= \\
\left(\left(\left(F_{1} \otimes F_{1}\right) \otimes F_{1}\right) \otimes\left(\left(F_{1} \otimes F_{1}\right) \otimes F_{1}\right)\right) \otimes\left(\left(\left(F_{1} \otimes F_{1}\right) \otimes F_{1}\right) \otimes\left(F_{1} \otimes F_{1}\right)\right),
\end{array}
$$

where we omitted a subscript 2 in $\otimes$ and also $u$ and $v$ that maximise the cardinalities of both intermediate and the top level product.

For calculation of the lower bound for function $h$ using Theorem 4, we need to calculate the values $(h(k))^{1 / k}$ for some positive integer $k$ which is as large as 
Table 2 The largest domains found for different $n$ using Algorithm 1

\begin{tabular}{lll}
\hline$n$ & Size & The structure of the largest domain found \\
\hline 2 & 2 & $F_{2}$ \\
3 & 4 & $F_{3}$ \\
4 & 9 & $F_{4}$ \\
5 & 20 & $F_{5}$ \\
6 & $F_{6}$ \\
7 & 45 & $F_{7}$ \\
8 & 100 & $F_{8}$ \\
9 & 222 & $F_{9}$ \\
10 & 488 & $F_{10}$ \\
11 & 1069 & $F_{11}$ \\
12 & 2324 & $F_{12}$ \\
13 & 5034 & $\left(F_{4} \otimes F_{3}\right) \otimes\left(F_{3} \otimes F_{3}\right)$ \\
14 & 10940 & $\left(F_{4} \otimes F_{3}\right) \otimes\left(F_{4} \otimes F_{3}\right)$ \\
15 & 24481 & $\left(F_{4} \otimes F_{4}\right) \otimes\left(F_{4} \otimes F_{3}\right)$ \\
16 & 54752 & $\left(F_{4} \otimes F_{4}\right) \otimes\left(F_{4} \otimes F_{4}\right)$ \\
17 & 123004 & $\left(\left(F_{4} \otimes F_{4}\right)\right) \otimes\left(\left(F_{3} \otimes F_{2}\right) \otimes F_{4}\right)$ \\
18 & 271758 & $\left.\left(\left(F_{3} \otimes F_{2}\right) \otimes F_{4}\right)\right) \otimes\left(\left(F_{3} \otimes F_{2}\right) \otimes F_{4}\right)$ \\
19 & 602299 & $\left(\left(F_{3} \otimes F_{2}\right) \otimes\left(F_{3} \otimes F_{2}\right)\right) \otimes\left(\left(F_{3} \otimes F_{2}\right) \otimes F_{4}\right)$ \\
20 & 1323862 & $\left(\left(F_{3} \otimes F_{2}\right) \otimes\left(F_{3} \otimes F_{2}\right)\right) \otimes\left(\left(F_{3} \otimes F_{2}\right) \otimes\left(F_{3} \otimes F_{2}\right)\right)$ \\
\hline
\end{tabular}

possible. Observing the Table 2, we note that the numbers $(h(k))^{1 / k}$ are strictly increasing with $k$ reaching 2.1045 for $k=20$.

Theorem 11 For large $n$, we have $h(n)>(2.1045)^{n}$.

Using Fishburn's Theorem 3, we can improve his lower bound for $f$

Theorem $12 f(n)>2.1890^{n}$ for all large $n$.

Proof Observing the Table 2, we note that the numbers $(h(k))^{1 / k-1}$ are strictly increasing with $k$ reaching 2.18909 for $k=20$.

It is interesting to note that, as Fishburn showed, the numbers $\left|F_{k}\right|^{1 / k-1}$ steadily decline starting from $k=12$. This is where the Fishburn's domains become not optimal.

\section{Upper bound}

We have much less information about upper bounds for functions $f, g, h$. It is known from Raz (2000) that there exists a constant $c>0$ such that $f(n)<c^{n}$. However, no such particular constant $c$ is known. Fishburn (1996a) conjectured (Conjecture 3) that the following inequality holds: 


$$
f(n+m) \leq f(n+1) f(m+1) .
$$

This conjecture, if proved, would imply $f(n) \leq 2.591^{n-2}$ (Fishburn 2002).

Danilov and Karzanov (2012) showed that there is a bijection between the set of maximal peak-pit Condorcet domains of maximal width and zonotopal tilings. The latter were shown to be in one-to-one correspondence with arrangements of pseudolines (see, for example, Chapter 6 in Felsner (2012)). The number of cutpaths of an arrangement of pseudolines is exactly the number of linear orders in a corresponding peak-pit domain of maximal width. Felsner and Valtr (2011) gave an upper bound for the number of cutpaths in an arrangements of pseudolines, which leads to the following theorem.

Theorem 13 For a sufficiently large n, we have $g(n)<2.4870^{n}$.

\section{Conclusion and further research}

Operations over Condorcet domains are useful in many respects. In particular, they lead to inequalities like (7) and (8) and eventually to lower bounds on the cardinality of largest Condorcet domains. The size of the largest Condorcet domain for a set of $n$ alternatives is of interest in several categories of Condorcet domains: all Condorcet domains, peak-pit domains and peak-pit domains of maximal width. Respectively, we are interested in the numbers $f(n), g(n)$ and $h(n)$ which output the cardinalities of largest Condorcet domains over $n$ alternatives in these categories.

In this paper, we improved the lower bound for function $f$, proved a lower bound for function $h$, and confirmed the lower bound for $g$ which was stated in the literature without proof. However, several important questions remain unanswered. It is highly likely but not yet known if $f(n)>g(n)$ for sufficiently large $n$. Another interesting problem, which remains, is getting upper bounds for these functions. It is known that $f(n)<c^{n}$ but no particular constant $c$ is known.

Acknowledgements The authors thank Dmitry Ignatov for computing assistance. Arkadii Slinko was supported by the Faculty Development Research Fund 3719899 of the University of Auckland. Alexander Karpov was partially supported by the Basic Research Program of the National Research University Higher School of Economics.

Funding Open Access funding enabled and organized by CAUL and its Member Institutions.

Open Access This article is licensed under a Creative Commons Attribution 4.0 International License, which permits use, sharing, adaptation, distribution and reproduction in any medium or format, as long as you give appropriate credit to the original author(s) and the source, provide a link to the Creative Commons licence, and indicate if changes were made. The images or other third party material in this article are included in the article's Creative Commons licence, unless indicated otherwise in a credit line to the material. If material is not included in the article's Creative Commons licence and your intended use is not permitted by statutory regulation or exceeds the permitted use, you will need to obtain permission directly from the copyright holder. To view a copy of this licence, visit http:// creativecommons.org/licenses/by/4.0/. 


\section{References}

Abello, J. (1991). The weak Bruhat order of $S_{\Sigma}$, consistent sets, and Catalan numbers. SIAM Journal on Discrete Mathematics, 4(1), 411-416.

Arrow, K. J. (1963). Social choice and individual values (2nd ed.). Wiley.

Black, D. (1948). On the rationale of group decision-making. Journal of Political Economy, 56(1), 23-34.

Danilov, V., Karzanov, A. \& Koshevoy, G. (2011). Condorcet domains of tiling type Condorcet domains of arXiv:1011.2888v2.

Danilov, V., Karzanov, A., \& Koshevoy, G. (2012). Condorcet domains of tiling type. Discrete Applied Mathematics, 160(7-8), 933-940.

Danilov, V., \& Koshevoy, G. (2013). Maximal Condorcet domains. Order, 30(1), 181-194.

Elkind, E. (2018). Restricted preference domains in social choice: Two perspectives. In X. Deng (Ed.), Algorithmic game theory (pp. 12-18). Springer International Publishing.

Elnitsky, S. (1997). Rhombic tilings of polygons and classes of reduced words in Coxeter groups. Journal of Combinatorial Theory, Series A, 77(2), 193-221.

Felsner, S. (2012). Geometric graphs and arrangements: some chapters from combinatorial geometry. Springer Science \& Business Media.

Felsner, S., \& Valtr, P. (2011). Coding and counting arrangements of pseudolines. Discrete and Computational Geometry, 46(3), 405.

Fishburn, P. C. (1996a). Acyclic sets of linear orders. Social Choice and Welfare, 14(1), 113-124.

Fishburn, P. C. (1996b). Decision theory and discrete mathematics. Discrete Applied Mathematics, 68(3), 209-221.

Fishburn, P. C. (2002). Acyclic sets of linear orders: A progress report. Social Choice and Welfare, 19(2), 431-447.

Galambos, A., \& Reiner, V. (2008). Acyclic sets of linear orders via the Bruhat orders. Social Choice and Welfare, 30(2), 245-264.

Kemeny, J. (1959). Mathematics without numbers. Daedalus, 88, 577-591.

Kemeny, J., \& Snell, L. (1960). Mathematical models in the social sciences. Ginn.

Kim, K., Roush, F., \& Intriligator, M. (1992). Overview of mathematical social sciences. The American Mathematical Monthly, 99(9), 838-844.

Labbé, J. P., \& Lange, C. (2020). Cambrian acyclic domains: Counting c-singletons. Order, 37, 571-603.

Leclerc, B., \& Zelevinsky, A. (1998). Quasicommuting families of quantum Plücker coordinates. Kirillov's Seminar on Representation Theory, 35, 85-108.

Le Marquise de Condorcet, M. (1785). Essai sur l'application de l'analyse à la probabilité des décisions rendues à la pluralité des voix. Paris.

Li, G., Puppe, C., \& Slinko, A. (2021). Towards a classification of maximal peak-pit Condorcet domains. Mathematical Social Sciences, 113, 191-202.

Monjardet, B. (2006). Condorcet domains and distributive lattices. Annales du LAMSADE, 6, $285-302$.

Monjardet, B. (2009). Acyclic domains of linear orders: A survey. In S. Brams, W. Gehrlein, \& F. Roberts (Eds.), The mathematics of preference, choice and order (pp. 139-160). Springer Berlin Heidelberg.

Mulder, H. M. (1978). The structure of median graphs. Discrete Mathematics, 24, 197-204.

Puppe, C. (2016). The single-peaked domain revisited: A simple global characterization. KIT Working Paper Series in Economics No. 97.

Puppe, C. (2018). The single-peaked domain revisited: A simple global characterization. Journal of Economic Theory, 17(6), 55-80.

Puppe, C., \& Slinko, A. (2019). Condorcet domains, median graphs and the single-crossing property. Economic Theory, 67(1), 285-318.

Raz, R. (2000). VC-dimension of sets of permutations. Combinatorica, 20(2), 241-255.

Slinko, A. (2019). Condorcet domains satisfying Arrow's single-peakedness. Journal of Mathematical Economics, 84, 166-175.

Zhan, P. (2019). A simple construction of complete single-peaked domains by recursive tiling. Mathematical Methods of Operations Research, 90, 477-488.

Publisher's Note Springer Nature remains neutral with regard to jurisdictional claims in published maps and institutional affiliations. 